\title{
Una mirada hacia la inclusión desde las representaciones sociales de los actores educativos
}

A view towards inclusion from the social representations of educational actors

\section{Aurora Elizabeth Montes Yacsahuache}

Lic. Educación Primaria

Mgtr. En Problemas de Aprendizaje

Docente de aula, I.E. 2060

amontesy@ucvvirtual.edu.pe, montesaurora846@gmail.com

https://orcid.org/0000-0003-2690-7973

https://scholar.google.es/citations?hl=es\&user=qsnXJooAAAAJ

\section{Carlos Enrique Bernardo Zárate}

Maestro en Docencia Universitaria

Universidad Nacional Federico Villarreal

carlosbernardpusmp@gmail.com

https://orcid.org/0000-0001-6960-6826

https://scholar.google.es/citations?user=9jGlQd8AAAAJ\&hl=es

\section{Claudia Noemi Rivera Rojas}

Doctora en Educación

Universidad Privada Cesar Vallejo

missclaudiariverar@gmail.com

https://orcid.org/0000-0001-7265-2727

https://scholar.google.com/citations?user=6MSjZ14AAAAJ\&hl=en

\section{Benita Sonia Mancilla Curi}

Licenciada en Educación Inicial

Maestro en Didáctica de la Enseñanza de la Educación Inicial - Universidad Peruana

Cayetano Heredia

Docente Educación Inicial Educación Básica - Docente Universidad Enrique Guzmán y

Valle bmancilla@ucvvirtual.edu.pe

bmancilla@une.edu.pe

https://orcid.org/0000-0002-4552-8090

Google académico: https://scholar.google.es/citations?user=BWFYGY0AAAAJ\&hl=es 
Abstract: The objective of this qualitative research was to know the social representations of educational actors about the inclusion of students with disabilities in Regular Basic Education Institutions. The Method was a qualitative, descriptive and interpretive study based on social phenomenology, the technique used for data collection was the semistructured interview applied to teachers, parents and specialists. Results From the inductive analysis of the data, three a priori categories resulted, showing that the information handled by educational actors is scarce, which does not favor the collapse of the fields of social representation that indicate, among other paradigms, that people with disabilities cannot learn or develop skills like other children who do not have disabilities, which builds attitudes that are detrimental to the cognitive and emotional capacities of the student, affecting their learning and adaptation to the social environment. Therefore, it is concluded that those of teachers and parents have little information and this generates confusion and divided opinions regarding the permanence of this population in regular schools and finally the attitude can influence in a positive or detrimental way for students who present some type of disability. key words: Social Representations, educational inclusion, disability

Resumen: La presente investigación cualitativa tuvo como objetivo conocer y analizar las representaciones sociales de los actores educativos sobre la inclusión de estudiantes con discapacidad en una institución de Educación Básica Regular. La Metodología usada fue un estudio cualitativo, descriptivo, basado en la fenomenología descriptiva, la técnica usada para la recolección de datos fue, la entrevista semiestructurada aplicadas a docentes, padres y especialistas. Los Resultados Del análisis inductivo de los datos resultaron 
tres categorías apriorísticas, demostrando que la información que manejan los actores educativos es escasa, lo cual no favorece al derrumbe de los campos de representación social que indican entre otras paradigmas, que las personas con discapacidad no pueden aprender ni desarrollar habilidades como otros niños que no tienen discapacidad, lo que construye actitudes que van en detrimento de las capacidades cognitivas y emocionales del estudiante, afectando su aprendizaje y adaptación al entorno social. Por lo que se concluye que los docentes y padres tienen escasa información y esta genera confusión y opiniones divididas respecto a la permanencia de esta población en escuelas regulares y finalmente la actitud docente puede influenciar de manera positiva o perjudicial para los estudiantes que presenten algún tipo de discapacidad.

Palabras clave: Representaciones Sociales, inclusión educativa, discapacidad

\section{INTRODUCTION}

En las últimas décadas, en el mundo, américa Latina y sobre todo en el Perú han emergido grandes cambios en el ámbito escolar, como la inclusión educativa. La inclusión de estudiantes con discapacidad está respaldada por organismos internacionales como: La Convención de los Derechos del Niño (1990), apoyada por La Declaración de Salamanca (1994), la Unicef, La Unesco y la ONU, no significa que esta se dé de manera eficiente y acogedora en las instituciones de Educación Básica Regular como lo sustenta el último informe de UNESCO (2020) De ahí la importancia de investigar sobre cómo se está llevando la inclusión en las instituciones de educación básica regular y cuáles son las representaciones sociales de los actores involucrados, para conocer y analizar estas representaciones con el fin de que nos sirva como 
punto de partida desde la perspectiva institucional para la toma de decisiones y la mejora del servicio educativo, por consiguiente, de la sociedad.

Así, tras muchas reflexiones y diálogos en relación al derecho que tienen todos los seres humanos de ser provisto de una educación de calidad para todos y todas, partiendo del acceso de niños y niñas a las escuelas que provean calidad en el aprendizaje, el desarrollo de sus habilidades individuales y la atención a sus necesidades educativas (ONU, 2016 p. 7).

Según la OMS calcula que el $15 \%$ de toda la población, es decir unos mil millones de habitantes en todo el mundo, presentan algún tipo de discapacidad; lo preocupante, es que todas estas personas deben hacer frente a muchos obstáculos o barreras para educarse, conseguir empleo, hacer una vida social normal, es decir, deben enfrentar diversas dificultades para acceder a una sociedad en igualdad de condiciones, lo que suele ir en detrimento emocional del individuo (Organización Mundial de la Salud, 2017, s.p.).

(Hincapié, Duryea e Hincapié, 2019), expresaron que en América Latina y el Caribe 31 países de los 33, han confirmado su compromiso con la igualdad de atención para las personas que presentan discapacidades, a través de instrumentos jurídicos internacionales y nacionales a nivel educativo (p. 9). Así mismo, (Marchesi, Blanco y Hernández 2014) manifestaron que los estudiantes de Bolivia, Costa Rica y México, que oscilan entre los 7 y 12 años y que poseen alguna discapacidad, tienden a tener probabilidades menores a las de otros niños sin discapacidad, por lo cual aún existe una gran lejanía a la inclusión total de estos niños a escuelas regulares, puesto que dichos países cuentan con altas tasas de discriminación. Por otro lado, Chile muestra todo lo contrario, pues presenta altas tasas de inclusión y las diferenciaciones se hacen cada vez menores (p. 14). 
En Perú, existen leyes que amparan y protegen la igualdad de derechos en educación, principalmente la ley de Educación 28044 en su artículo $3^{\circ}$ declara que la educación es un derecho esencial de toda la persona y de la sociedad y el Decreto ley $\mathrm{N}^{\circ} 25762$, Ley orgánica del Ministerio de Educación a través de la dirección de Educación Básica Especial las cuales están comprometidas con la inclusión en la educación para las personas con discapacidad, estas leyes se consolidad en el respeto, la empatía y la tolerancia hacia los demás. Según el censo escolar realizado en el año 2019, se registra un total de 21 mil 157 instituciones de Educación Básica Regular, que cuentan con al menos un estudiante con Necesidades Educativas Especiales (NEE) matriculado, cifra que representa el 19,74\% del total de instituciones y encuentran dificultades para atenderlos adecuadamente. Mientras el 80,26\% de Instituciones de Educación Básica Regular no cuentan con estudiantes matriculados con NEE. En este sentido, es importante acotar el escaso presupuesto asignado a la Educación Básica Regular para la educación inclusiva, el cual apenas alcanza el $0,62 \%$ del presupuesto total del sector educación destinado a la contratación y capacitación docente de la educación inclusiva, materiales de infraestructura y acompañamiento, lo que limita la reducción de la brecha de exclusión en el sistema educativo de los estudiantes con NEE, asociadas a la discapacidad (Informe de la defensoría $N^{\circ} 183,2019$ ).

No obstante a ello, en un informe de la defensoría del pueblo emitido en el año 2019, afirma que en nuestro país Perú, se está incrementando la cantidad de estudiantes que presentan algún tipo de discapacidad en el sistema educativo; sin embargo, aún se visualizan actitudes negativas $\mathrm{y}$ discriminación durante el periodo de matrícula de los niños discapacitados en las instituciones regulares, lo que se traduce en la reducción significativa de la participación de la comunidad y los desempeños de aprendizaje (Defensoría del Pueblo, 2019). 
En este contexto, la incorporación de estudiantes con discapacidad o exigencias especiales busca la igualdad de oportunidades para todos y se apoya en el principio del respeto a la diversidad (Fernández y Duarte, 2016). Dicho respeto a la diversidad, se ha visto debilitado por las representaciones sociales, en relación a las personas que tienen alguna discapacidad (Urbina y Ovalles, 2018). Lamentablemente, la construcción de representaciones sociales hacia cierta población etiquetada como incapaz por el hecho de presentar alguna discapacidad, ha generado a nivel mundial una gran brecha y ha tenido una gran influencia en el ingenio colectivo, al haberse creado símbolos y significados sobre ellos, que los han venido condenando a la discriminación, dejándolos al margen de la formación y capacitación, lo que ocasiona una reducción de sus habilidades y posibilidades de desarrollo profesional (Rojas, 2017).

En este sentido, la concientización sociocultural frente a la discapacidad, es el punto de entrada hacia ciertas y antiguas formas de pensamiento $y$ estereotipos que se han sumado en el transcurso de la historia y a la cual se le ha asignado a cierto grupo de población, al calificarlos dado su discapacidad la cual es asociada a la incapacidad de pensar, de aprender, de actuar y de hacer. Estas descripciones acaban por afectar desfavorablemente el desarrollo educativo de las personas discapacitadas.

Por otro lado, los estudiantes que presentan discapacidad leve o moderada que son integrados a las instituciones de educación básica regular, no se les ofrece un servicio educativo de calidad, debido a que se omiten las adaptaciones curriculares que se ajusten a sus necesidades y no reciben el apoyo necesario por parte de los profesionales, por falta de personal y de recursos (Defensoría del Pueblo, 2019).

Ante lo expuesto y lo concreto, de lo ideal plasmado en normas y leyes a la realidad práctica del día a día en las aulas regulares, era necesario hacer una 
indagación de las representaciones sociales con respecto a la inclusión educativa para conocer las construcciones por parte de los actores educativos en lo que concierne a la inclusión de personas con discapacidad en la escuela básica regular, para lo cual nos planteamos la siguiente interrogante ¿Cuáles son las representaciones sociales de los actores educativos frente a la inclusión de estudiantes con discapacidad en una institución de educación básica regular? Esta incógnita sirvió como eje conductor con el cual se orientó la investigación.

Así mismo; esta investigación fue motivada a partir de la percepción de actitudes no apropiadas dirigidas a cierta población que presentaba características diferentes en una institución educativa por lo que se sostiene que esta investigación es importante y tiene relevancia social ya que buscó conocer, analizar y develar las Representaciones Sociales llámese, conocimientos, creencias, actitudes, etc. a partir del discurso de los actores educativos, para analizarlas y tener una visión de la realidad.

Existe un gran vacío respecto a este tema, muchas investigaciones se han realizado a nivel internacional y nacional en función a las representaciones sociales de un actor educativo y en la gran mayoría se han abocado a la percepción de los docentes, por lo que esta investigación permite dar una mirada más holística y a partir del análisis de varios actores educativos llámese maestros, padres de familia y especialistas, permitió obtener una visión más amplia sobre el tema que de manera silenciosa avanza sin que se tome medidas al respecto. Así mismo desde el análisis de las representaciones sociales de los actores educativos respecto a la inclusión de estudiantes con discapacidad aportó conocimiento de la realidad existente para tomar decisiones que mejoren las condiciones de una buena implementación sobre la inclusión educativa. 
Como objetivo general tenemos Conocer las representaciones sociales de los actores educativos sobre la inclusión de estudiantes con discapacidad en una Institución de Educación Básica Regular. Teniendo como objetivos específicos: Analizar la información sobre la inclusión de estudiantes con discapacidad en un aula regular a partir del discurso de los actores educativos. Analizar los campos de representación sobre la inclusión de estudiantes con discapacidad en un aula regular a partir del discurso de los actores educativos y analizar la actitud sobre la inclusión de estudiantes con discapacidad en un aula regular a partir del discurso de los actores educativos.

\section{Materiales y métodos}

Esta investigación se enmarcó dentro de un enfoque cualitativo el cual estudia los fenómenos sociales y humanos, que, para (Cerrón, 2019) la investigación cualitativa es una investigación flexible, sistemática y crítica del comportamiento de los agentes educativos. Con un diseño fenomenológico descriptivo el cual intentó describir las experiencias vividas, y una vez obtenido los datos se procedió al análisis de los mismos a través de la reducción fenomenológica (Giorgi, 2009).

La técnica aplicada en esta investigación fue la entrevista semi estructurada, la cual permitió recolectar la información en base a preguntas efectuadas a los participantes. Esta técnica tuvo como ventaja mejorar las preguntas en cuanto se identificaba la inserción de una o la mejora de otra, ello en función de esta característica que la distingue. (De acuerdo a Hernández y Mendoza 2018), en la aplicación de esta técnica se requiere la intervención de dos personas que tratan sobre un tema en particular y existe una interrelación entre ellos mediante preguntas y respuestas.

El instrumento utilizado fue la Guía de entrevista la cual constó de 10 preguntas y permitió acceder al discurso de los actores educativos en función a las representaciones sociales sobre la inclusión de estudiantes con 
discapacidad y direccionadas al rescate de la información en función a las categorías apriorísticas, que es analizar la información sobre la inclusión de estudiantes discapacitados, en cuanto al campo de representación analizar el significado y creencias respecto a la discapacidad y finalmente analizar la actitud de los actores educativos sobre la discapacidad de estudiantes. El tiempo de duración de la entrevista por cada actor educativo fue entre 10 y 22 minutos.

Los participantes en el siguiente estudio de investigación constituyeron los docentes de aula, especialistas de SAANEE y las madres de familia de los estudiantes inclusivos de la institución educativa 2060 de la UGEL 04. El número de participantes fueron nueve, tres docentes de aula, todas mujeres que se encuentran entre los 50 y 55 años de edad, con más de veinte años de servicio y de estado civil casadas. Tres madres de familia que están entre los 22 y 33 años de edad, de las cuales dos sólo llegaron a culminar la secundaria y una realizó estudios superiores en un instituto técnico, todas son convivientes. Y tres especialistas de SAANEE que se encuentran entre los 45 y 55 años de edad, con más de veinticinco años de servicio, de estado civil casadas.

Los criterios de selección de los participantes fueron: docentes nombrados y que cuenten con estudiantes inclusivos, se excluyó a todos los docentes contratados y también aquellos docentes nombrados que no tengan en sus aulas estudiantes inclusivos. así mismo se consideró a las madres de familia que tengan hijos que presenten alguna discapacidad y que estén dispuestas a manifestar sus percepciones, conocimientos y experiencias respecto a la inclusión de estudiantes con necesidades especiales en escuelas regulares El tamaño de la muestra fue por muestreo intencionado no probabilístico ya que los participantes de estudio fueron tomados según los criterios de selección establecidos, que para el criterio de (Hernández, 2015) afirma que 
el número de participantes dependerá de los criterios relacionados con las características de la investigación o el propósito del investigador lo que permitirá tomar decisiones. Así mismo cuanto más pequeño sea el número de la muestra, mayor será su credibilidad (Patton, 1990). Este muestreo te permite seleccionar casos específicos con una determinada característica de la población y en la que el escenario de población es muy variable (Otzen \& Manterola 2017).

\section{Resultados}

Los resultados fueron asumidos partiendo de un análisis descriptivo de la información recopilada de las entrevistas aplicadas que fueron en total nueve, las cuales se enfocaron en tres grupos de personas, tres especialistas de educación inclusiva, tres docentes de aula y tres madres de familia que tiene hijos con discapacidad, estudiantes de la escuela regular. Cuyos resultados se representan mediante tres categorías apriorísticas, de las cuales se disgregan nueve subcategorías.

Los resultados generales demuestran que la información que manejan los actores educativos es escasa, lo cual no favorece al derrumbe de los campos de representación social que indican entre otras paradigmas, que las personas con discapacidad no pueden aprender ni desarrollar habilidades como otros niños que no tienen discapacidad, lo que construye actitudes que van en detrimento de las capacidades cognitivas y emocionales del estudiante, afectando su aprendizaje y adaptación al entorno social.

Con respecto al conocimiento acerca de la inclusión de estudiante con discapacidad en escuelas regulares a partir del discurso de los actores educativos, se evidencia que los especialistas de SAANEE manejan conocimiento al respecto están relacionados a las políticas, prácticas y cultura educativa para poder brindar pertenencia y calidad en los aprendizajes de los niños, respetando y fomentando el desarrollo de la diversidad, considerando 
que todos los niños discapacitados cuentan con habilidades diferentes y las cuales deben ser potencializadas a través del sistema educativo.

Mientras que, en el caso de los docentes, pese a que consideran que la educación inclusiva, busca el trato igualitario y sin discriminación para todos los niños que presenta discapacidad, a través de lo cual se espera que estos puedan adaptarse y desarrollar integralmente conforme sus habilidades diferentes, dentro del sistema educativo regular, promoviendo su desarrollo para el futuro. Manifiestan que han tenido que atender muchos casos de niños con problemas de lenguaje, con déficit intelectual, por tanto, el conocimiento adquirido depende básicamente de la experiencia de cada caso, sin embargo, destacan que no han sido capacitados y formados al respecto, según la discapacidad que tenga el niño inclusivo.

Las madres de familia manejan muy poca información acerca de la educación inclusiva; lo que constituye una debilidad en cuanto a la exigencia de los derechos fundamentales de los niños con discapacidad, dando lugar a que en algunos casos sean rechazados con excusas o pretextos que no son válidos y que no van en desacuerdo con lo establecido en ley. Lo que ha generado que muchos niños, han tenido que comenzar muy tarde en el colegio, lo que desfavorece su proceso de aprendizaje.

En este sentido, se analiza que existe una brecha entre el conocimiento que manejan los especialistas, los docentes y los padres de familia en cuanto a la inclusión educativa; lo cual no favorece el desarrollo exitoso de la educación inclusiva dado que siendo de ellos, que depende los resultados de la inclusión, la información y niveles debería ser igualitario para propiciar sus aportes y participación en la adaptación del niño discapacitado y la promoción de sus habilidades diferentes. Esta diferencia en cuanto al conocimiento puede ser evidenciada en las respuestas captadas durante la entrevista donde los especialistas demuestran su sensibilización y aceptación de las políticas 
inclusivas, los docentes tienen conocimiento general y algunas madres de familia no están muy claras de que significa la inclusión y a quienes va dirigida.

“Tengo conocimiento en cuanto a las políticas, las prácticas y la cultura educativa para poder ofrecer una calidad educativa con pertinencia $y$ eficacia para los estudiantes que presenten discapacidad y diversidad, por tanto, las instituciones están abiertas las puertas para que puedan ingresar los estudiantes de la diversidad y la discapacidad" (Especialista 2).

"Conozco que la educación inclusiva responde a un desafío que se nos presenta de forma individual con cada niña inclusiva que debemos atender y los cuales tienen habilidades diferentes" (Docente 3).

"Es de los que tienen retardo mental, también hay sordos y mudos" (Madre 3)

Por otra parte, la Percepción acerca del rechazo que tienen las personas acerca de los estudiantes con discapacidad en escuelas regulares a partir del discurso de los actores educativos. Se refiere a la impresión y consideración que tiene los individuos en relación a inclusión de los estudiantes con discapacidad a las escuelas regulares.

En relación a ello, los especialistas consideran que el rechazo hacia las personas con discapacidad viene arraigado al temor y la falta de conocimiento en cuanto a la manera de cómo afrontar la discapacidad de los niños incluidos, por lo que es un tema que va más allá de la percepción o de discriminación, sino de conocimiento para poder diseñar estrategias pedagógicas que puedan esquematizar métodos de trabajo que sean apropiados según el caso que se presente dentro del aula de clase. Tomando en consideración que cada estudiante con o sin discapacidad presenta habilidades diferentes y, por tanto, debe tener una atención a sus necesidades individuales 
En el caso de los docentes, opinaron que el rechazo a las personas con discapacidad representa una actitud errada que básicamente es fundamentada en la desinformación, falta de conocimiento e insensibilización en cuanto a la situación y a lo que conlleva el hecho de tener una discapacidad. En muchos casos el rechazo viene dado por el temor de no poder afrontar el reto de la inclusión, por no sentirse académicamente bien empoderado, disponer de tiempo y comodidad por seguir trabajando igual, sin hacer cambios para poder gestionar adecuadamente la inclusión y avance en el aula de un niño con discapacidad.

Mientras que las madres, opinan que ante el rechazo que tienen algunas personas hacia los niños discapacitados, tienen sentimientos encontrados que van desde la rabia hasta el llanto, al ver la poca sensibilización que tienen algunas personas al momento de hacer comentarios fuertes de sus hijos y ver la reacción de sus pequeños, los daños psicológicos y emocionales que esto puede ocasionar considerando que el trabajo de ellas como madre, es arduo especialmente a nivel de lograr la estabilidad emocional de sus hijos como fortaleza principal para hacer frente a su discapacidad.

En consideración al análisis descriptivo de esta subcategoría se puede corroborar que los actores educativos coinciden en referir que el rechazo hacia las personas con discapacidad es el resultado de la falta de información, de conocimiento, de sensibilización y de miedo hacia aquello que desconocen y no encuentran como enfrentar. Consideran que las personas pueden llegar a ser crueles con sus comentarios al respecto y que no son empáticos. Además de ello, algunos actores educativos tienen la premisa de que el estudiante con discapacidad no va a adquirir aprendizaje por lo que asumen que los esfuerzos serán en vano. 
"Bueno yo creo que esa situación se da por falta de conocimiento, porque realmente, creo que todos deberíamos tener la oportunidad de demostrar las capacidades que uno tiene" (Especialista 1).

"Pienso que, para algunos docentes, no es de su agrado la educación inclusiva pues lo toman como una recarga de trabajo que a la final no da resultados, pero pienso que no está bien, porque todos los estudiantes tienen derecho a tener la misma educación que los demás estudiantes regulares y creo que la actitud no es la correcta” (Docente 1).

"En mi caso me creó bastante angustia y cólera, que incluso la misma directora me rechazara el ingreso del niño al colegio, porque el espacio es muy reducido, me pusieron muchos pretextos por lo que me sentí emocionalmente muy mal" (Madre 1).

Estos resultados corroboran una vez más la triste realidad que está pasando en nuestro país respecto a la inclusión de estudiantes con discapacidad y lo que sucede en el mundo como lo sostiene la UNESCO (2020) con el último informe de Revisiones de Mejoramiento de la Educación de Perfiles (PEER) muestra que muchos países siguen practicando la segregación en la educación, lo que refuerza los estereotipos, la discriminación y la alienación. Al analizar los campos de representación sobre la inclusión de estudiantes con discapacidad en aulas regulares a partir del discurso de los actores educativos. Los campos representacionales comprenden todos los significados que se le asignan al objeto de representación y que pueden ser diversos, como juicios, afirmaciones, tipologías, creencias y elementos culturales que lo construyen. En cuanto al significado para los actores educativos del ingreso de los estudiantes con discapacidad en las escuelas de educación básica regular. Comprende la idea o representación que el individuo tiene en relación a los estudiantes con discapacidad y su ingreso en las escuelas que imparten educación básica regular 
Los especialistas indicaron que para ellos, la inclusión educativa de las personas con discapacidad significa el respeto al derecho universal de la educación, el rechazo a la discriminación, la integración y aceptación de una sociedad que es diversa, el respeto a la identidad propia y a un modelo social que se ajusta a una sociedad con valores y sensibilizada ante una realidad única, una conceptualización del ser humano que puede ser útil y productivo conforme sus habilidades y destrezas particulares, las cuales deberán ser desarrolladas desde los primeros años de vida con el apoyo de los docentes y de los padres de familia.(E1,E2,E3)

Los docentes consideran que los estudiantes dependiendo de la discapacidad que presenten, algunos niños deben ir a escuelas especiales donde puedan recibir la atención que ellos ameritan y puedan estar en grupos de trabajo más reducidos que en un aula regular, donde el docente por más que quiera, tiene una carga de estudiantes que superan los 35 alumnos, lo cual no favorece el tiempo y dedicación que necesita un estudiante inclusivo. (D2, D3)

Todas las madres entrevistadas están de acuerdo que lo ideal es que los niños con discapacidad asistan a colegios regulares, porque perciben que de esta manera se apoya su aprendizaje y su interacción social, haciendo que ellos se sientan parte del entorno en el cual viven y se sientan capaces y seguros como cualquier otro niño regular. (M1, M2)

En cuanto a las Representaciones Sociales de los actores educativos se evidencia opiniones contradictorias entre los entrevistados, pues pese a que reconocen el derecho que tienen todos los niños discapacitados a acceder a la educación, algunos consideran que dependiendo de la discapacidad deben tener una atención especializada para su propio beneficio, pues consideran que tenerlos en aulas regulares pueden ser contraproducente para la evolución de su aprendizaje y desarrollo de habilidades, ello como resultado de la falta 
de conocimiento especializado, de recursos y de tiempo para atender algunos caso donde la discapacidad sea más severa.

Así mismo acerca de la creencia sobre la discapacidad como una limitación para el aprendizaje de un estudiante, todos los actores coinciden que las discapacidades de los niños no limitan su aprendizaje, pues se ha corroborado que cada uno tiene sus habilidades diferentes y que pueden desarrollar con éxito muchas actividades, la limitación está en el apoyo que se le da dentro del aula de clases, las estrategias pedagógicas y didácticas que diseñe el docente para cada estudiante, ajustándose a sus necesidades educativas y potenciando sus habilidades diferentes e inclusivas. Lo cual debe ir de la mano con el apoyo de familia, desde el hogar los cuales deben ser partícipes de todo el proceso de aprendizaje.

En concordancia con los resultados obtenidos se puede afirmar que las Representaciones Sociales de los actores respecto a la discapacidad que presentan los estudiantes señalan que, no es ninguna limitación para el aprendizaje, tal como lo menciona (Kirby, 2017), que, la educación inclusiva en Perú debe aún enfrentar muchos estereotipos o representaciones sociales, como por ejemplo; las personas con discapacidad no cuenta con la capacidad de aprender, lo cual es totalmente falso, dado que los estudios demuestran que todos los niños aprenden a ritmos distintos o niveles diferentes, aun cuando no presentan una discapacidad, por tanto la educación inclusiva defiende la teoría de que no se debe buscar que todos aprendan lo mismo ni al mismo ritmo, pues cada uno cuentan con habilidades diferentes.

Con respecto al lugar de formación educativa de los estudiantes discapacitados. Esta subcategoría consiste en el lugar o sitio donde consideran los diferentes actores educativos que han sido entrevistados deben ir los niños que presentan discapacidad para poder obtener su formación educativa. En este caso, los especialistas consideran que los niños deben ser previamente 
evaluados, porque dependiendo de la severidad de la discapacidad en algunos casos es mejor que los niños sean ingresados al CEBE, no por discriminarlos o aislarlos, sino porque en estas instituciones tienen los mecanismos, recursos y formación más adecuada para su atención especializada a diferencia de los colegios regulares.

En coincidencia a ello, los docentes señalan que todos los niños con discapacidad deben ir a colegios especiales no porque están en desacuerdo con la educación inclusiva sino porque ellos no son formados o capacitados para atender estas necesidades educativas, por lo que muchos sienten que es un reto que no pueden asumir con posibilidades de éxito. Por su parte las madres, comprenden que, si el beneficio de sus hijos discapacitados amerita una atención especializada, preferirían que fueran ingresados en escuelas especialmente diseñadas para ello, donde los puedan ayudar mejor con su aprendizaje, pese a que consideran a que apoyan la educación inclusiva.

En análisis a los resultados descriptivos sobre la actitud que se tiene sobre la inclusión de estudiantes con discapacidad en las aulas regulares, se indica que las actitudes de los docentes afectan positivamente en los niños cuando se trata de docentes empáticos, que tiene un trato igualitario y que se preocupan por atender las necesidades educativas de los estudiantes inclusivos; mientras que en caso contrario, cuando se trata de docentes que tienen una actitud negativa y estar predispuesto hacia el rechazo de los niños con discapacidad, afectan su estabilidad emocional lo que genera que el niño se aísle del grupo, no desee asistir al colegio y no esté motivado para el desarrollo de las actividades académicas, acentuando la diferenciación entre los niños con discapacidad y aquellos que no presentan ninguna discapacidad, lo cual es totalmente contrario a lo que se desea lograr con la educación inclusiva.

Se observa también que la actitud frente a las personas con discapacidad va a depender de las Representaciones Sociales que tengan los actores educativos, 
de la información, el conocimiento, la experiencia, la sensibilización y la empatía que tengan las personas acerca de la discapacidad y la inclusión educativa. Lo que va a estar determinado por las representaciones sociales, que en la mayoría de los casos son las principales barreras para el desarrollo de la educación inclusiva.

\section{Discusión}

En cuanto a la información sobre la inclusión de estudiantes con discapacidad en aulas regulares a partir del discurso de los actores educativos se tiene que, el conocimiento que se maneja en relación a la inclusión hace la diferencia entre la actitud, percepción y aceptación de los estudiantes con discapacidad. En el caso de los especialistas conocen el propósito, los beneficios, las necesidades de la inclusión y el tratamiento que debe darse, además de que conocen las leyes, distribución o asignación de los niños inclusivos según su discapacidad y nivel de severidad, por lo que están más sensibilizados con el tema y obtiene mejores resultados, demostrando más compromiso y vocación de servicio; sin embargo, se evidencian varias debilidades en los docentes en cuanto a la información y conocimiento que manejan en relación a la educación inclusiva, su capacidad para diseñar estrategias educativas, la formación que tiene en relación a cómo integrar a un estudiante con discapacidad a las actividades académicas, como lograr su aprendizaje significativo, captar su atención y potencializar sus habilidades diferentes. Lo que genera rechazo, miedo y resistencia al cambio que propone el sistema de educación inclusiva.

Las madres tampoco manejan mayor información en la educación inclusiva, e incluso desconocen muchos aspectos importantes en relación a la discapacidad de sus hijos. Lo que motiva que muchas tengan más preocupación y angustia de lo normal, ante la situación desconocida que 
deben enfrentar día tras día con sus niños discapacitados. En general se pueden mencionar que las únicas personas, que manejan información en relación a la educación inclusiva, son los especialistas como resultado de su formación y su experiencia en instituciones como CEBES, especializadas en la atención con niños con habilidades diferentes, sin embargo, tanto los docentes como las madres que son los actores educativos que están más relacionados con los niños con discapacidad presentan muchas debilidades que son las barreras para el desarrollo del aprendizaje de los niños inclusivos. Esta representación social permite una brecha que afecta el desarrollo del estudiante inclusivo dentro del aula de clases, porque es el docente el que está en su día a día. Esta situación es muy similar a la referida por Gallego y Otero (2020) en su investigación quienes señalaron que los docentes no tienen formación en cuanto al modelo de la educación inclusiva, lo que ha traído como consecuencia que adopten dentro de las aulas un modelo integrador, lo que afecta su capacidad para el diseño y formulación de estrategias inclusivas que mejoren el nivel de enseñanza y con ello favorezca el desarrollo académico del estudiante inclusivo.

Todos los actores educativos, cuentan con experiencias en casos de discapacidad, sin embargo, en ocasiones los docentes deben enfrentar a discapacidades en la cual no han tenido experiencia previa y por ende, carecen de conocimiento suficiente para atender los diferentes tipos de discapacidades; al igual que las madres que vienen formándose de forma autodidacta con el paso del tiempo, terapias y atenciones dirigidas a sus hijos discapacitados; pero no tienen una formación técnicas sino de forma empírica, similar a la de los docentes. Esta experiencia de los actores educativos (docentes, especialistas y padres de familia) en cuanto a la discapacidad, tiene repercusión en el desarrollo integral de los niños discapacitados; tal como lo menciona Urbina y Ovalles (2018) quien indicó 
que la participación del docente es fundamental en la inclusión de estudiantes discapacitados, especialmente en escuelas regulares de educación básica.

Parte de la desinformación y ausencia de conocimiento en cuanto a la educación inclusiva, ha propiciado los paradigmas que tienen algunas personas en cuanto a las habilidades y capacidad de aprendizaje de los niños discapacitados. Esta situación presenta para los actores educativos, una percepción negativa con una actitud que es desfavorable para el desarrollo y alcance de competencias de la educación inclusiva. Lo cual según lo señalado por Gajardo y Torrego (2020), los docentes que tiene experiencias previas en relación a la inclusión, suelen ser más receptivos y ver los aspectos más humanos y emocionales del individuo, colocándolos por encima del contexto netamente académico. Lo cual coincide con lo indicado por Rojas (2017) quien concluyó que la representación social u conceptualización hacia el estudiante con discapacidad se inicia en el panorama y óptica del docente, en una perspectiva errónea frente a un estudiante con discapacidad, sólo por poseer particularidades personales que no son comunes en el sistema educativo.

Por otro lado, la inclinación que tiene el docente porque el estudiante con discapacidad asista a un colegio especializado viene asociado a representaciones sociales que lo llevan a pensar que los estudiantes discapacitados no tienen la capacidad de aprender, al desconocimiento de otros mecanismos de aprendizaje fuera de lo tradicional y la poca disponibilidad por asumir el reto de la inclusión. Estas representaciones sociales y/o prácticas segregadoras se siguen aplicando como lo confirma el último informe de la Unesco (2020) la cual señala que muchos países siguen practicando la segregación en la educación, lo que refuerza los estereotipos, la discriminación y la alienación, donde el $42 \%$ de los países tienen leyes que 
exigen que los estudiantes con discapacidades sean educados en entornos separados.

Las actitudes de los docentes, los especialistas, las madres de familia y el resto de los miembros de la comunidad educativa, es fundamental para el progreso del aprendizaje de los estudiantes discapacitados, para propiciar su adaptación al sistema educativo, su interacción social, el fortalecimiento de su seguridad y confianza y por ende sus aspectos cognitivos. Cuando un docente no es empático y demuestra rechazo hacia su estudiante con habilidades diferentes, este inmediatamente captará estas actitudes negativas y afectará emocionalmente al estudiante, lo que se traduce en efectos negativos a nivel cognitivo y de conducta en el estudiante, lo que limitará su integración al entorno social y académico. Esta situación podría deteriorar la autoestima del estudiante y de su seguridad como persona, para actuar y reaccionar ante situaciones complejas a nivel académico y personal

$\mathrm{Si}$ bien es cierto que todos los actores educativos coinciden en que los estudiantes deben asistir a una escuela regular como parte del derecho que le corresponde como persona, es aquí donde las Representaciones Sociales se tornan un poco ambiguas ya que madres como docentes, preferirían que estos asistan a una escuela especializada y así lo sustenta Jigyel, et al. (2018). En su artículo percepciones de los padres sobre la educación inclusiva para estudiantes con NEE, en la cual los padres señalan que la inclusión de sus hijos en escuelas regulares mejoró en gran parte el peso del cuidado diario, y estaban contentos, ellos guardaban una gran preocupación porque observaban conductas inadecuadas, en algunas veces acoso y escaso aprendizaje académico, por lo que estos referían la necesidad de que los docentes de aula obtengan asesoramiento y capacitación continua.

Estos resultados coinciden con Barreiro \& Zambrano (2020) quienes sostienen que es evidente la necesidad que tienen estas personas con 
discapacidad ya que son vulneradas por la sociedad al existir barreras que no les permiten la participación en distintos eventos, como, sociales, políticos y laborales. Y que van a requerir de la intervención de un trabajador social quien con su actuar busca minimizar dichas situaciones.

Así mismo Romero \& Vergara (2017) reafirman coincidentemente que existe este problema al sostener que no existe un modelo evaluativo para estudiantes con necesidades especiales, que garantice sus derechos y esto debido a que no existe personal especializado para la atención de esta población, la falta de conocimientos de los docentes para la atención a estos estudiantes, el gran número de estudiantes por aula y el desinterés de los docentes por asumir la atención a estudiantes con necesidades educativas especiales, hace que se perciba que este es un problema persiste y no se está haciendo nada por solucionarlo.

Sin embargo, este problema podría persistir como lo mencionan otros autores y que dado los resultados al conocer las representaciones sociales de los actores educativos se evidencia que existe una escasa aplicación de una metodología activa en el proceso educativo de los niños y esto se debe a que los maestros no se actualizan en su formación con capacitaciones permanentes, ni tampoco toman en cuenta el material didáctico que permite que el aprendizaje sea motivador y significativo tanto para el proceso normal como para el inclusivo. (Bravo et.ál 2019)

Dentro de las limitaciones del presente estudio encontramos la falta de estudios previos en la que se involucre a varios actores educativos para conocer su discurso a partir de las representaciones sociales, del mismo modo se han encontrado trabajos con otros diseños como el hermenéutico y muy poco de R,S. con una fenomenología descriptiva

\section{Conclusiones}


Primera. Las representaciones sociales que poseen los actores educativos, llámese, especialistas, docentes de aula y madres de familia respecto a la inclusión de estudiantes con discapacidad pueden parametrar o favorecer la inclusión de estudiantes en la medida en que sus demandas de conocimiento, formación, capacitación logren sensibilización y empoderamiento en la labor educativa.

Segunda. Existe una brecha entre la información que manejan los especialistas, los docentes y los padres de familia en cuanto a la inclusión educativa; lo cual no favorece el desarrollo exitoso de la educación. Los especialistas demuestran mayor sensibilización y aceptación a las políticas inclusivas, los docentes tienen conocimiento general y algunas madres de familia no están muy claras de que significa la inclusión y a quienes va dirigida. Sin embargo; todos los actores educativos involucrados en la investigación tienen experiencia con este grupo de población respecto a la convivencia, más no en la atención adecuada como respuesta educativa a las necesidades de aprendizaje.

Así mismo se puede concluir que las representaciones sociales que poseen los actores educativos respecto a la información sobre la inclusión de estudiantes discapacitados coinciden, al referir, que el rechazo hacia las personas con discapacidad es el resultado de la falta de información, de conocimiento, de sensibilización y de miedo hacia aquello que desconocen y no encuentran como enfrentar. Además de ello, algunos actores educativos tienen la premisa de que el estudiante con discapacidad no va a adquirir aprendizaje por lo que asumen que los esfuerzos serán en vano. Esta desinformación y falta de formación ha traído como consecuencia el temor y las debilidades en cuanto al diseño de estrategias adecuadas a nivel pedagógico respecto a los niños discapacitados, lo que va en detrimento al éxito de la educación inclusiva. 
Tercera. Se evidencia opiniones contradictorias entre los actores educativos pues pese a que reconocen el derecho que tienen todos los niños discapacitados a acceder a la educación, algunos consideran que deben tener una atención especializada para su propio beneficio, pues consideran que tenerlos en aulas regulares pueden ser contraproducente para la evolución de su aprendizaje y desarrollo de habilidades, ello como resultado de la falta de conocimiento especializado, de recursos y de tiempo para atender algunos casos donde la discapacidad sea más severa. Así mismo todos los actores educativos coinciden que las diferentes discapacidades de los estudiantes no limitan el aprendizaje de los mismos, que cada uno posee habilidades diferentes y pueden llegar a realizar exitosamente muchas actividades, en todo caso la discapacidad es una condición y no una limitación.

Cuarta. Se concluye que la actitud frente a las personas con discapacidad puede depender de la información, el conocimiento, la experiencia, la sensibilización y la empatía que tengan las personas acerca de la discapacidad y la inclusión educativa. Lo que va a estar determinado por las representaciones sociales, que en la mayoría de los casos son las principales barreras para el desarrollo de la educación inclusiva.

\section{Referencias}

Barreiro, L. \& Zambrano, D. (2020). Trabajo Social y Discapacidad: Intervención desde el departamento de calificación del Ministerio de salud Pública. Socialium. 4. 181-192. https://doi.org/10.31876/s1.v4i2.86

Bravo, N., Guerrero, E., Espinosa, A. G. \& Espinosa, F. J. (2019). El desarrollo de la educación inicial y su implicación en los recursos didácticos. Revista Tecnológica Ciencia y Educación Edwards Deming. 3. 67-80. https://doi.org/10.37957/ed.v3i2.36 
Cerrón, W. (2019). La investigación cualitativa en educación. Horizonte De La Ciencia, 9(17), 1-8. Recuperado a partir de http://167.114.2.69/index.php/horizontedelaciencia/article/view/219

Defensoría del Pueblo (2019). En la Libertad el 88\% de personas con discapacidad no tienen acceso al sistema educativo. Lima, Perú. Recuperado de https://www.defensoria.gob.pe/en-la-libertad-el-88-de-personas-condiscapacidad-no-tienen-acceso-al-sistema-educativo/.

Fernández, F. y Duarte, J. (2016). Retos de la inclusión Académica de Personas con Discapacidad en una Universidad Pública Colombiana. Formación universitaria, 9(4), 95104. https://dx.doi.org/10.4067/S0718-50062016000400011

Gajardo, K. y Torrego, L. (2020). Representaciones sociales sobre inclusión educativa de una nueva generación docente. Revista Educación, política y sociedad vol. 5(1), pp. 11-38. Universidad de Valledolid. España. Recuperado de ISSN 2445-4109.

Gallego, G. y Otero, L. (2020). Hacía la educación inclusive y personalizada: opinines e ideario educativo del profesorado. Universidad Internacional de la Rioja. Revista de educación inclusive vol. 4 (1), pp. 47-70. España. Recuperado de https://core.ac.uk/download/pdf/289266274.pdf

Giorgi, A. (2009). The Descriptive Phenomenological Method in Psychology: A Modified Husserlian Approach. Duquense. Recuperado de:https://www.academia.edu/33413268/The_Descriptive_Phenomenologic al_Psychological_Method

Hernández A. (2015). Representaciones sociales de docentes de secundaria sobre la inclusión de estudiantes sordos en el aula regular. Revista Virtual Universidad Católica del Norte, 46, 102-114 Recuperado de http://revistavirtual.ucn.edu.co/index.php/RevistaUCN/article/view/702/122 
Hernández-Sampieri, R. \& Mendoza, C. P. (2018). Metodología de la investigación: las rutas cuantitativa, cualitativa y mixta. México: McGraw Hill.

Hincapié, D., Duryea, S., \& Hincapié, I. (2019). Education for All: Advancing Disability Inclusion in Latin America and the Caribbean. Banco Interamericano de Desarrollo. https://doi.org/10.18235/0001673

Jigyel, K., Miller, A., Mavropoulou, S., \& Berman, J. (2018). Benefits and concerns: parents' perceptions of inclusive schooling for children with special educational needs (SEN) in Bhutan. International Journal of Inclusive Education, 24(10), 1064-1080. https://doi.org/10.1080/13603116.2018.1511761

Kirby, M. (2017). Implicit assumptions in special education policy: Promoting full inclusion for students with learning disabilities. Child \& Youth Care Forum, 46(2), 175-191. http://dx.doi.org/10.1007/s10566-016-9382-x

Marchesi, A.; Blanco, R.; \& Hernández, L. (2014). Avances y desafíos de la educación inclusiva en Iberoamérica. Madrid: OEI.

ONU (2016). XI y XII Jornadas de Cooperación Educativa con Iberoamérica sobre Educación Especial e Inclusión Educativa. Recuperado de: https://www.educacionyfp.gob.es/dam/jcr:35ac31ee-c2e6-4835-962fc7d01e757103/xi\%20y\%20xii\%20jornadas\%20de\%20cooperacion\%202010-16.pdf

Organización Mundial de la Salud (2017). 10 datos sobre la discapacidad. Recuperado de https://www.who.int/features/factfiles/disability/es/

Otzen, T. \& Manterola, C. (2017). Técnicas de muestreo sobre una población a estudio. Int. J. Morphol., 35(1):227-232, 2017. http://dx.doi.org/10.4067/S0717-95022017000100037

Patton, M. (1990). Qualitative evaluation and research methods (pp. 169186). Beverly Hills, CA: Sage. 
Riaño, M. \& Triviño, N. (2019). Alfabetizar, una experiencia pedagógica llena de sentidos. Revista Tecnológica Ciencia y Educación Edwards Deming. 3. 15-40. https://doi.org/10.37957/ed.v3i2.33

Rojas, M. (2017). Representaciones sociales de los docentes de Educación Primaria sobre la inclusión de escolares con discapacidad. Fundación Dialnet Vol. 3 (1), pp. 49-86. Recuperado de https://dialnet.unirioja.es/servlet/articulo?codigo=6296631

Romero, C. \& Vergara, D. (2017). Por una evaluación incluyente: el principio de una educación equitativa. Perspectivas, (5), 22-33. Recuperado a partir de https://revistas.uniminuto.edu/index.php/Pers/article/view/1576

Formatos de citación

UNESCO (2020). Revisiones de Mejoramiento de la Educación de Perfiles (PEER). Recuperado de: https://education-profiles.org/

Urbina, J. y Ovalles, G. (2018). Teoría de las representaciones sociales. Una aproximación al estado del arte en América Latina. Psicogente Vol. 21(40), pp. 495-544. https://doi.org/10.17081/psico.21.40.3088.

Villamar, M. \& Moreira, T. (2020). Familia como espacio de resiliencia en las personas con capacidades diferentes de la Facultad de Ciencias Humanísticas y $\quad$ Sociales. Socialium. 4. 417-428. https://doi.org/10.31876/sl.v4i2.92 